\title{
Thermal characteristics study of light aggregate concrete of the middle Atlas pozzolana
}

\section{Etude des caractéristiques thermiques de bétons de granulats légers de la pouzzolane du moyen Atlas}

\author{
A. Bouyahyaoui ${ }^{1}$, M. Cherkaoui ${ }^{1,2},{\text { My L. } \text { Abidi }^{1}, \text { T. } \text { Cherradi }}^{1}$ \\ ${ }^{1}$ Département de Génie Civil, EMI, Université Mohamed V, Rabat, Maroc \\ ${ }^{2}$ Ecole Nationale Supérieure des Mines de Rabat (ENSMR), Rabat, Maroc
}

\begin{abstract}
We present in this work an experimental study of the thermomechanical characterization of pozzolan (volcanic rock widespread in the region of Azrou in the Middle Atlas in Morocco). The objective of this work is to evaluate the effect of the pozzolan on the thermal conductivity of pozzolanic concrete by using different concrete formulations and this, by substituting various percentages of gravel and usual sands with gravel and sand of the pozzolan. The thermal properties of the samples consist to determine the thermal conductivity and the heat capacity. The results obtained show that the pozzolan is a local material with a high thermal resistance and can be used as a good insulated material in heat transfer and energy efficiency in the building.
\end{abstract}

Résumé : Dans ce travail, nous présentons une étude expérimentale portant sur la caractérisation thermomécanique de la pouzzolane (roche volcanique très répandue dans la région d'Azrou au Moyen Atlas au Maroc). L'objectif de ce travail est d'évaluer l'influence de la pouzzolane sur la conductivité thermique du béton pouzzolanique en utilisant différentes formulations de béton et ce, en substituant des pourcentages variés de graviers et sables usuels par des graviers et sables à base de la pouzzolane. La caractérisation thermique des échantillons a consisté à déterminer la conductivité thermique et la capacité calorifique. Les résultats obtenus ont montré que la pouzzolane est un matériau local qui présente une résistance thermique importante et peut être considérée comme un bon isolant en efficacité énergétique et thermique dans le secteur du bâtiment.

\section{Introduction}

Dans le cadre de la transition énergétique, la réglementation thermique de la construction marocaine appliquée depuis 2015, exige que les déperditions dans le bâtiment n'excèdent pas un certain seuil selon le zonage climatique du pays [1-2]. Pour se conformer à ce règlement, deux possibilités peuvent être envisagées, soit d'augmenter les épaisseurs des éléments de l'enveloppe et des séparations, soit de travailler avec des matériaux de faible conductivité thermique. La première solution reste difficile à appliquer pour les maisons de petite surface vu qu'elle entraine une consommation importante d'espace et à fortiori un prix de revient du produit très couteux. De ce fait, la deuxième solution semble être plus acceptable. Dans cette optique, nous proposons d'étudier un matériau de construction élaboré à base de pouzzolane.
La pouzzolane est une roche volcanique du moyen Atlas marocain qui se définit comme un matériau silicoalumineux. Elle se caractérise par sa légèreté et sa grande porosité qui est de l'ordre de $45 \%$ [2].

L'objectif de notre travail est de confectionner un béton et un mortier contenant de la pouzzolane et d'évaluer et comparer leurs caractéristiques thermophysiques (la conductivité thermique et la capacité calorifique) à celles du béton et du mortier usuels.

\section{Etude expérimentale}

Notre étude expérimentale a consisté en l'étude de la conductivité thermique et la capacité calorifique d'échantillons à base de pouzzolane. 


\subsection{Mesure de la conductivité thermique: méthode du plan chaud}

Plusieurs méthodes sont utilisées pour la mesure de la conductivité thermique des matériaux, il s'agit notamment de [3-4], [5-6] :

Plaque Chaude gardée ;

Méthode Fluxmétrique ;

Méthode Flash ;

Fil Chaud ;

Plan Chaud ;

Source Plane Transitoire ;

Source plane modifiée.

Vu la nature de notre matériau et la disponibilité des appareils de mesures, notre calcul a été effectué par la méthode du plan chaud [7-8].

Les échantillons étudiés ont été confectionnés en substituant un pourcentage des graviers usuels par des graviers pouzzolaniques, et une partie du sable de mer par du sable pouzzolanique

Pour la simplification, nous avons utilisé les abréviations suivantes :

- BnGPZ: Béton contenant $\mathrm{n} \%$ des graviers pouzzolaniques à la place des graviers usuels.

- BnSPZ: Béton contenant $\mathrm{n} \%$ du sable pouzzolanique à la place du sable de mer.

- MnSPZ: Mortier contenant $\mathrm{n} \%$ du sable pouzzolanique à la place du sable de mer.

Les mélanges étudiés sont: B25GPZ, B40GPZ, B100GPZ, B40SPZ, M50SPZ et M100SPZ.[2-3]

Les échantillons ont été préparés selon les dimensions normalisées des films chauds, à savoir :

- une forme cylindrique: rayon $=10 \quad \pm 0,4 \mathrm{~cm}$ et épaisseur $=2 \pm 0,4 \mathrm{~cm}$

- une forme parallélépipédique : $10 \times 10 \times 2 \mathrm{~cm} 3$

Etant donné que la conductivité thermique est influencée par l'humidité, les échantillons ont été placés dans l'étuve à $70^{\circ} \mathrm{C}$ jusqu'à ce que leur masse se stabilise.

\section{Technique de mesure}

La méthode est basée sur la création d'un flux de chaleur unidirectionnel passant par l'échantillon et le polystyrène à l'aide d'une résistance thermique. Le montage expérimental se compose des éléments suivants :

Un polystyrène, isolant placé au-dessous de la résistance thermique, permet de maximiser le flux passant à travers l'échantillon.

Deux blocs d'aluminium, utilisés pour réduire le temps de la phase transitoire profitant de sa grande conductivité.

Trois thermocouples pour la mesure de la température dans des points particuliers (Fig. 1.).

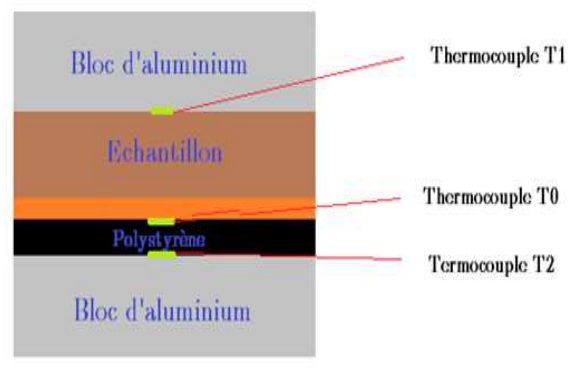

Fig. 1. Méthode du plan Chaud

En effectuant le bilan énergétique de l'échantillon :

Flux induit par effet de Joule

$\phi_{\mathrm{J}}=\mathrm{RI}^{2}=\mathrm{R} \times\left(\frac{\mathrm{U}}{\mathrm{R}}\right)^{2}=\frac{\mathrm{U}^{2}}{\mathrm{R}}$

Avec

$\mathrm{U}$ : tension aux bornes de la résistance chauffante $(\mathrm{V})$

$\mathrm{R}$ : Résistance chauffante $(\Omega)$

Flux de conduction traversant l'échantillon

$\phi_{\mathrm{E}}=\frac{\lambda_{1} \mathrm{~S}}{\mathrm{e}_{1}}\left(\mathrm{~T}_{0}-\mathrm{T}_{1}\right)$

Avec

$\lambda_{1}$ : La conductivité thermique de l'échantillon

$S$ : L'aire de l'échantillon

$\mathrm{e}_{1}$ : Epaisseur de l'échantillon

T0 : Température de la plaque chauffante

T1: Température entre l'échantillon el le bloc d'aluminium

Flux de conduction traversant le polystyrène

$\phi_{\mathrm{P}}=\frac{\lambda_{2} \mathrm{~S}}{\mathrm{e}_{2}}\left(\mathrm{~T}_{0}-\mathrm{T}_{2}\right)$

Avec

$\lambda_{2}:$ La conductivité thermique du polystyrène

$\mathrm{S}: \mathrm{L}$ 'aire du polystyrène

$\mathrm{e}_{2}$ : Epaisseur du polystyrène

T0 : Température de la plaque chauffante

T2: Température entre le polystyrène et le bloc d'aluminium

En utilisant les équations (1), (2) et (3), on trouve que :

Alors

$$
\phi_{\mathrm{J}}=\phi_{\mathrm{E}}+\phi_{\mathrm{P}}
$$

$$
\frac{\mathrm{U}^{2}}{\mathrm{R}}=\frac{\lambda_{2} \mathrm{~S}}{\mathrm{e}_{2}}\left(\mathrm{~T}_{0}-\mathrm{T}_{2}\right)+\frac{\lambda_{1} \mathrm{~S}}{\mathrm{e}_{1}}\left(\mathrm{~T}_{0}-\mathrm{T}_{1}\right)
$$

Finalement

$$
\lambda_{1}=\frac{\mathrm{e}_{1}}{\mathrm{~T}_{0}-\mathrm{T}_{1}}\left[\frac{\mathrm{U}^{2}}{\mathrm{RS}}-\frac{\lambda_{2}}{\mathrm{e}_{2}}\left(\mathrm{~T}_{0}-\mathrm{T}_{2}\right)\right]
$$

\subsection{Mesure de la capacité calorifique de la pouzzolane en poudre : Méthode du calorimètre différentiel}

La capacité thermique massique à pression constante $\mathrm{Cp}$, est l'une des grandeurs thermodynamiques les plus importantes. Elle permet de déterminer les variations d'enthalpies en fonction de la température pour une phase 
donnée, le pouvoir de stockage de l'énergie thermique par la chaleur sensible et de déterminer implicitement la diffusivité et l'effusivité thermique d'un matériau.

La pouzzolane a été broyée sous forme de poudre pour la préparation des échantillons de $21 \mathrm{mg}$.

\section{Technique de mesure}

L'appareil utilisé, TA Instrument Q100, constitué de deux enceintes chauffantes et identiques, l'une contient l'échantillon à analyser et l'autre la référence (fig. 2 et fig. 3). Lors de l'utilisation de la DSC (Calorimétrie Différentielle à Balayage), une augmentation linéaire de la température est imposée ; on enregistre alors la différence de puissance électrique $\mathrm{W}(\mathrm{t})$ fournie aux deux enceintes (fours) afin de les maintenir en permanence à une température identique $(\mathrm{T} 1(\mathrm{t})=\mathrm{T} 2(\mathrm{t}))[9-10]$

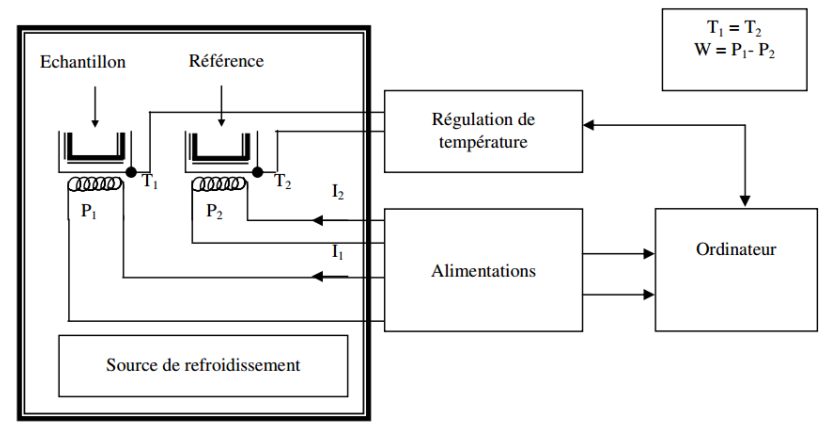

Fig. 2: Principe de mesure du calorimètre différentiel

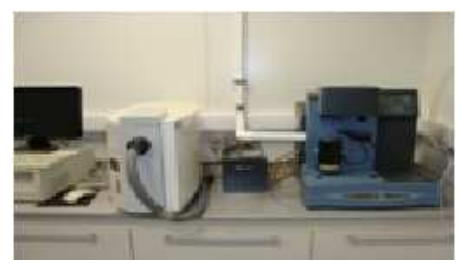

Fig.3. Calorimètre différentiel

Principe de la détermination du $\mathbf{C p}$

L'énergie fournie au corps

$Q=\Delta \mathrm{H}=\mathrm{m} \mathrm{C}_{p} \Delta \mathrm{T}$

$\frac{d H}{d t}=\mathrm{mC}_{p} \frac{d T}{d t}$

$\frac{d H}{d t}$ : correspond au flux de chaleur que mesure la DSC

$\frac{d T}{d t}$ : correspond à la vitesse de montée en température réelle lors de l'essai

$$
\text { Donc } \mathrm{C}_{p}=\frac{\frac{d H}{d t}}{\mathrm{~m} \frac{d T}{d t}}
$$

\section{Résultats}

Les résultats de l'étude de la conductivité thermique des échantillons sont résumés dans les tableaux cidessous :

Table 1. Conductivité thermique du béton pouzzolanique \begin{tabular}{|c|c|}
\hline Echantillon & Conductivité \\
\hline
\end{tabular}

\begin{tabular}{|l|c|}
\hline & thermique (W/K.m) \\
\hline B25GPZ & 0,985 \\
\hline B40GPZ & 0,808 \\
\hline B100GPZ & 0,528 \\
\hline B40SPZ & 1,229 \\
\hline
\end{tabular}

Table 2. Conductivité thermique du mortier pouzzolanique

\begin{tabular}{|c|c|}
\hline Mélanges & $\begin{array}{c}\text { Conductivité } \\
\text { thermique (W/K.m) }\end{array}$ \\
\hline M50SPZ & 0,506 \\
\hline M100SPZ & 0,289 \\
\hline
\end{tabular}

Nous constatons que l'incorporation du gravier pouzzolanique dans le béton permet de réduire la conductivité thermique. Elle passe de $0,985 \mathrm{~W} / \mathrm{K}$.m pour un béton contenant $25 \%$ de gravier pouzzolanique (B25GPZ) à $0,528 \mathrm{~W} / \mathrm{K} . \mathrm{m}$ pour un béton contenant uniquement du gravier à base de pouzzolane (B100GPZ) (Table 1).

De même pour le mortier, la conductivité thermique est passée de $0,506 \mathrm{~W} /\left({ }^{\circ} \mathrm{K} . \mathrm{m}\right)$ pour M50SPZ à 0,289 $\mathrm{W} /\left({ }^{\circ} \mathrm{K} . \mathrm{m}\right)$ pour M100SPZ (Table 2$)$.

En ce qui concerne la capacité calorifique, nous remarquons qu'elle augmente en fonction de la température. Elle passe de 765,7 (J/K.Kg) pour une température de $-10^{\circ} \mathrm{C}$ à $1096(\mathrm{~J} / \mathrm{K} . \mathrm{Kg})$ pour une température de $290^{\circ} \mathrm{C}$ (Fig. 4)

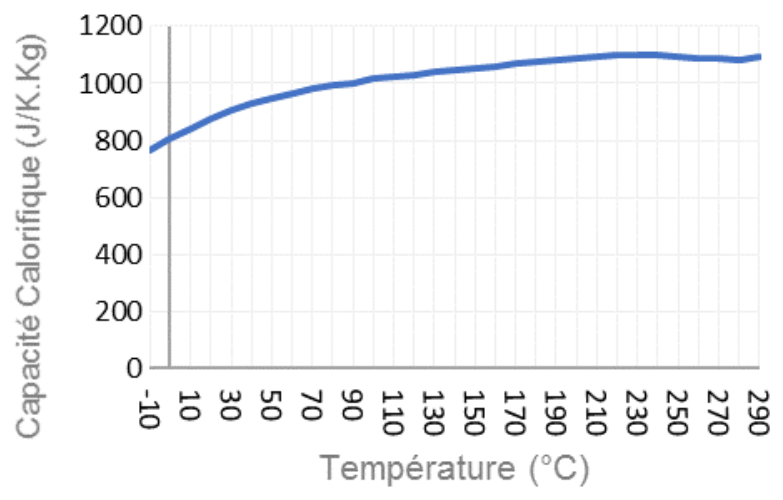

Fig. 4. Capacité calorifique en fonction de la température.

\section{Discussion}

La pouzzolane se caractérise par une grande porosité, due aux nombreuses bulles d'air qu'elle contient. Son incorporation dans le béton et le mortier permet de réduire la conductivité thermique. Cette réduction est de l'ordre de $76 \%$, elle passe de $2,2 \mathrm{~W} /\left({ }^{\circ} \mathrm{K} . \mathrm{m}\right)$ pour un béton usuel à $0,528 \mathrm{~W} /\left({ }^{\circ} \mathrm{K} . \mathrm{m}\right)$ pour un béton à gravier entièrement à base de pouzzolane. Pour le mortier, elle diminue d'environ $78 \%$. Elle passe de $1,3 \mathrm{~W} /\left({ }^{\circ} \mathrm{K} . \mathrm{m}\right)$ pour un mortier usuel à $0,289 \mathrm{~W} /\left({ }^{\circ} \mathrm{K} . \mathrm{m}\right)$ pour un mortier à sable entièrement à base de pouzzolane; ce résultat est conforme aux études bibliographiques [11].

En ce qui concerne la capacité calorifique, elle est de l'ordre de $900 \mathrm{~J} /{ }^{\circ} \mathrm{K} . \mathrm{Kg}$ pour des températures d'utilisation dans le secteur du bâtiment $\left(-10^{\circ} \mathrm{C}\right.$ à $\left.45^{\circ} \mathrm{C}\right)$. Ce qui est en en concordance avec les résultats de la littérature [12]. 
Le comportement mécanique de ces mélanges a montré une faible résistance à la compression par rapport au béton usuel ce qui limite leur utilisation aux éléments secondaires non structuraux [2].

L'introduction de ces matériaux dans la couche de forme et la couche de protection de l'étanchéité permet d'amortir la variation de la température de la face interne et d'augmenter le déphasage entre les pics des températures.

\section{Conclusion}

La pouzzolane du moyen Atlas peut jouer un rôle très important dans la politique de la transition énergétique. Son utilisation permet de réduire les déperditions thermiques à travers les éléments de l'enveloppe du bâtiment : les ponts thermiques et la couche d'enduit des façades internes et externes (le mortier).

Enfin, il est recommandé d'étudier le volet économique de ces solutions et de les comparer à l'utilisation d'un isolant thermique existant sur le marché.

\section{Références}

1. Réglementation thermique de la construction marocaine RTCM, Novembre 2015

2. A. Bouyahyaoui, M. Cherkaoui, My L. Abidi, T.Cherradi Mechanical and chemical characterisation of pozzolan of middele atlas in morocco accepted, International Journal of GEOMATE, Jan., 2018 Vol.14, Issue 41, pp.126-134

3. B. Ranaivo Rabehaja, Etude des caractéristiques thermique et mécaniques de béton de pouzzolane, Thèse de doctorat, université paris 7, décembre 1986

4. A. Degiovanni, M. Laurent., Nouvelle technique d'identification de la diffusivité thermique pour méthode flash, Journal of Applied Physics, Vol. 21, n'3, pp. 229-237, 1986.

5. Y. Jannot, P. Meukam, Simplified estimation method for the determination of thermal effusivity and thermal conductivity with a low cost hot strip, Measurement Science and Technology, Vol. 15, pp. 1932-1938, 2004.

6. S.E. Gustafsson, Transient plane source techniques for thermal conductivity and thermal diffusivity measurements of solid materials, Rev. Sci. Instrum., Vol. 62, pp.797- 804, 1991.

7. Sébastian Gauthier, Caractérisation thermique de la matière par la méthode $3 \omega$, thèse de doctorat Université Languedoc Roussillon 2012

8. N. OZISIK : Heat conduction. John Wiley, Sons inc ., 1993.

9. Jean Grenet, Bernard Legendre, Analyse calorimétrique différentielle à balayage (DSC) techniques de l'ingénieur 10 déc. 2010

10. H.G.E. Hohne, W.F Hemmnger Differential scanning calomerty. Springer 2003

11. S.Azizi, C.Moyne, A.Degiovanni Experimental And Theoretical Approach Of The Thermal Conductivity Of Wet Porous Media International Journal Of Heat
And Mass Transfer Volume 31, Issue 11, November 1988, Pages 2305-2317

12. P. Meukam, Y.Jannot, A. Noumowe, T.Ckofane Thermo Physical Characteristics Of Economical Building Materials Construction And Building Materials Volume 18, Issue 6, July 2004, Pages 437443 